\title{
Effect of valproate versus levetiracetam monotherapy on reproductive functions in newly diagnosed epileptic males
}

\author{
Wafaa S. Mohamed ${ }^{1}$, Rania S. Nageeb ${ }^{1 *}$ D, Noha A. Hashim ${ }^{1}$ and Alaa A. Omran²
}

\begin{abstract}
Background: Antiepileptic drugs are responsible for reproductive dysfunction in male epileptic patients.

Objectives: To evaluate the effect of valproate (VPA) versus levetiracetam (LEV) as a monotherapy on males' sex hormones and sperm parameters in newly diagnosed epileptic patients.

Materials and methods: This comparative study included 50 newly diagnosed epileptic male patients. All patients were subjected to complete general and neurological examination, Doppler ultrasonography of the testis, pre- and post-treatment serum sex hormone assay, and semen analysis. Post-treatment re-evaluation checkpoint was determined if 8 weeks have passed after the last seizures. This study included two groups, group I (25 patients were treated by VPA) and group II (25 patients were treated by LEV).

Results: There was no statistical difference between groups regarding their baseline characteristics. In group I, at post-treatment checkpoint, we found significant decrease in luteinizing hormone and follicle-stimulating hormones and highly significant increase of prolactin serum level. In sperm parameters, there was significant decrease of all measured items. In group II, serum sex hormone was not significantly changed at post-treatment checkpoint but semen analysis parameters were significantly decreased regarding all items. In comparison between the two groups, there was significant decrease of sperm concentration among group II with highly significant decrease of normal morphology of sperm among group I.
\end{abstract}

Conclusion: Both VPA and LEV had adverse effects on the semen of male epileptic patients while VPA had also an adverse effect on sex hormones.

Keywords: Epilepsy, Antiepileptic drugs, Males, Reproduction

\section{Background}

Epilepsy is a chronic neurological disorder characterized by recurrent epileptic attacks, and researches have reported that the relationship between epilepsy and the reproductive dysfunction is not yet completely understood [1]. Seizures are linked with sexual dysfunction as part of the disease pathophysiology in these patients [2]. Through epileptic discharge, the hypothalamus receives signals from the hippocampus, amygdala, and cerebral cortex. Hypothalamic-pituitary axis signaling changes is leading

\footnotetext{
* Correspondence: rnsanad@yahoo.com

'Department of Neurology, Faculty of Medicine, Zagazig University, Zagazig,

Sharkia, Egypt

Full list of author information is available at the end of the article
}

to sex hormone release including follicle stimulating hormones (FSH) and luteinizing hormone (LH) changes [3].

However, the available antiepileptic drugs (AED) have not yet been proven to have negative impacts on sexual function. Evaluation of AED-induced sexual dysfunction is complicated, less than clear, and is hard to find. Sex hormone levels can be changed due to AEDs which are the cause of sexual dysfunction and reproductive disorders [4].

Among these antiepileptic drugs is valproate (VPA) which is a commonly used, broad-spectrum antiepileptic drug that is very effective in the treatment of different types of generalized and partial seizures [5]. Also, levetiracetam (LEV) is a second generation, broad-spectrum antiepileptic drug which is commonly used. LEV has high efficacy, 
safety, and favorable profile with low incidence of adverse effects and drug-drug interactions in all age groups [6].

VPA may affect epilepsy patients' hormones and this may result in reproductive problems. VPA may reduce sperm motility, increase the frequency of morphologically abnormal sperm, and be associated with small testicular size in men with epilepsy [7]. Also animal studies concerning the effects of LEV on sex hormones concluded that LEV might affect steroid hormone secretion. However, human studies yielded diverse results [2].

To the best of our knowledge, there have been no previous humane studies comparing the effects of valproate and levetiracetam on the male reproductive system, as both of them is commonly used in male patients of reproductive age. Therefore, the current study was designed to evaluate the effect of valproate versus levetiracetam as a monotherapy on males' sex hormones and sperm parameters in newly diagnosed epileptic males.

\section{Materials and methods}

The present study was a comparative type. All patients were selected from the outpatient neurology clinic of Zagazig University Hospitals, Sharkia Governorate, Egypt, in the period from June 2016 to March 2017, in the department of Neurology Zagazig University Hospitals. Samples were collected by the systematic random method. The study included 50 newly diagnosed epileptic males (clinically and electrophysiologically ((EEG)) according to the International League Against Epilepsy ((ILAE)) classification 2010) [8].

\section{Sample size calculation}

To detect this size effect in the analysis of variance between the two examined drug groups with two repeated measurements (basal and eight weeks after seizure control) at 0.05 alpha level of significance, a sample size of 25 subjects per group was found to achieve more than 95\% power. Sample size was calculated using Epi Info version seven [9].

\section{Inclusion criteria}

All patients were enrolled if their ages are between 18 and 45 years. All patients had normal brain imaging (Brain CT or MRI). Also, all patients had normal thyroid functions and normal sex hormones assay (luteinizing hormone, follicle-stimulating hormones prolactin, testosterone, and estradiol).

Patients are currently receiving hormonal replacement therapy or any drugs that may affect reproductive functions were excluded. Other criteria for exclusion were patients with significant or progressive medical illness; patients with testicular atrophy, varicocele, or infectious diseases were also excluded from the study.

All patients were subjected to thorough history taking of seizure semiology, complete general and neurological examination, and pre- and post-antiepileptic drug treatment serum sex hormones assay and semen analysis. Doppler ultrasonography of the testis was performed for all patients to exclude the diagnosis of undetected testicular atrophy varicocele and infectious diseases.

\section{Laboratory investigations}

Fasting morning (between 7:00 a.m. and 8:00 a.m.) venous blood samples have been withdrawn in the morning before and after treatment at outpatient's clinic of Zagazig University Hospitals, Clinical Pathology laboratories. These morning venous samples were centrifuged. After $20 \mathrm{~min}$, the serum was separated and the samples were frozen at $-20^{\circ} \mathrm{C}$ until hormone assays were performed for the following reproductive hormones:

Luteinizing hormone (LH) (normal range men 1.7$8.6 \mathrm{mIu} / \mathrm{mL}$ )

Follicle-stimulating hormones (FSH) (normal range $1.5-12.4 \mathrm{mIu} / \mathrm{mL})$

Prolactin (PRL) (normal range men 4.04-15.2 ng/mL)

Testosterone (T) (normal range men $2.8-8 \mathrm{ng} / \mathrm{mL}$ )

Estradiol (E2) $(7.63-42.6 \mathrm{pg} / \mathrm{mL})$

Semen samples have been obtained through masturbation after 3-6 days of abstinence and examined for semen parameters. After liquefaction, the samples were evaluated for sperm concentration, count, motility, and morphology using a computer-assisted semen analysis (Sperm Class Analyzer CASA System, MICROPTIC Spain). The system follows WHO (2010) strict criteria for the assessment of human semen.

Patients were subclassified into two groups who were matched in age, group I (25 patients) was treated by valproate (VPA) as an initial monotherapy for seizures. VPA dosage was adjusted to maintain plasma concentration of $70-80 \mu \mathrm{g} / \mathrm{mL}$ [10]. Group II that included $25 \mathrm{pa}$ tients was treated by levetiracetam (LEV) as a monotherapy. Our patients underwent gradual dose increment (starting from 500 to $1000 \mathrm{mg} /$ day) for either VPA or LEV till they become seizure-free at the last 8 weeks before post-treatment checkpoint.

Post-treatment re-evaluation point was determined if 8 weeks have passed after the last seizure attacks, and if seizures occurred, we waited for another 8 weeks [2], that is, to exclude the effect of epileptic discharges on sex hormones [7].

Daily dose was calculated by dividing the total dose by duration of treatment by days. Treatment duration was calculated by the time (weeks) passed between the preand post-treatment checkpoint.

\section{Statistical analysis}

Qualitative data were presented as number $(N)$ and percentage (\%). Quantitative data were expressed as mean 
$(M) \pm$ standard deviation (SD). Chi-square test $\left(\chi^{2}\right)$ was used to compare between two qualitative variables. $t$ test was used to detect the relationship between two quantitative variables (groups). All the statistical tests were done using statistical package of social science (SPSS version, 22). $P$ values $<0.05$ were considered significant, and $P$ values $<0.001$ were considered highly significant [11].

\section{Results}

Fifty male patients who had a new diagnosis of idiopathic generalized or partial epilepsy were included in the present study. Patients were subclassified into two groups who were matched in age, group I (25 patients) was treated by valproate as an initial monotherapy for their seizures. Group II that included 25 patients was treated by levetiraceram as a monotherapy. Their mean ages $( \pm$ SD) were $30.6( \pm 10.5)$ and $31.8( \pm 10.1)$ years, respectively.

In comparison between group I and group II regarding baseline characteristics, we did not find any statically difference between them regarding age, seizure type, treatment duration, or dose $(P<0.05)$ (Table 1$)$.

Regarding valproate-treated patients (group I), in comparison between pre- and post-treatment checkpoint, we found statistical significant decrease in LH and FSH $(P=$ $0.01)$ and high statistical significant increase of prolactin $(P<0.001)$ with non-significant decrease in testosterone and estradiol $(P>0.05)$. However, all sperm parameters at post-treatment checkpoint were statistically decreased especially total sperm motility $(p=0.029)$ (Table 2$)$.

As regard to levetiracetam-treated group (group II), we found that male sex hormones were decreased at posttreatment checkpoint that did not reach statistical significant difference $(P>0.05)$. But, regarding the comparison between pre- and post-treatment of sperm parameters among group II, there was significant decrease in all measured items $(P=0.01)$ that was a high significant difference at total sperm motility one $(P=<0.001)$ (Table 3$)$.

In comparison between group I and group II at posttreatment checkpoint regarding sperm parameters, we found non-significant difference between them regarding sperm motility or count with significant decrease of sperm concentration among group II with highly significant decrease of normal morphology of sperm among group I (Fig. 1).

\section{Discussion}

Among male epileptic patients, gonadotropic hormone dysregulation, semen parameter impairment, decreased in fertility, and sexual dysfunctions have been reported. From the probable causes was the effect of antiepileptic drugs [12].

In the present study, valproate-treated group (group 1) was examined pre- and post-treatment checkpoint; we found a significant decrease in serum LH and FSH at post-treatment checkpoint. This was in accordance with Xiaotian and colleagues [10]. This decrease in LH and FSH levels credited to negative feedback regulation in the pituitary portal system [13] as well as valproate affects the metabolism of serotonergic and gamma-aminobutyric acid (GABA)-ergic steroids or by a direct effect of VPA on endocrine tissue, in particular in the testis which is supported by studies in dogs, rats, and goats [13, 14].

We found non-significant decrease in serum total testosterone $(\mathrm{T})$ levels at post-treatment checkpoint among valproate-treated group, and this was in accordance with Roste and colleagues [14] and Mikkonen and colleagues [15] who found no significant difference in testosterone level in epileptic patients treated by valproate who compared with the healthy males as a control group.

However, Rattya and colleagues [13] stated that valproate could increase serum androgen concentration involving testosterone in male epileptic patients. Najafi and colleagues [16] showed that valproate-treated males had significantly lower mean testosterone level. Also, Hamed and colleagues [17] in their study found that men with epilepsy had lower serum levels of free testosterone and higher sex hormone-binding globulin (SHBG). Free bioactive testosterone represents $2 \%$ of total testosterone, and the majority of plasma testosterone is linked to albumin and SHBG. Increased SHBG would expect to produce sexual dysfunction by decreasing serum levels

Table 1 Baseline characteristics of the studied groups

\begin{tabular}{|c|c|c|c|c|c|c|c|c|}
\hline \multicolumn{2}{|c|}{$\underline{\text { Variable }}$} & \multicolumn{2}{|c|}{ Group I (VAP), $n=25$} & \multicolumn{2}{|c|}{ Group II (LEV), $n=25$} & \multirow{2}{*}{$\frac{T}{0.41}$} & \multirow[t]{2}{*}{$x^{2}$} & \multirow{2}{*}{$\frac{P \text { value }}{0.68}$} \\
\hline Age & $\begin{array}{l}(\mathrm{M} \pm \mathrm{SD}) \\
\text { Range (years) }\end{array}$ & $\begin{array}{l}30.6 \\
18-3\end{array}$ & & $\begin{array}{l}31.8 \\
20-4\end{array}$ & & & & \\
\hline \multicolumn{2}{|c|}{ Seizure type } & No & $(\%)$ & No & (\%) & & & \\
\hline \multicolumn{2}{|c|}{ - Partial } & 5 & $(20.0)$ & 10 & $(40.0)$ & 2.86 & & 0.23 \\
\hline \multicolumn{2}{|c|}{ - Partial with secondary generalization } & 7 & $(28.0)$ & 7 & $(28.0)$ & & & \\
\hline \multicolumn{2}{|c|}{ - Generalized } & 13 & $(52.0)$ & 8 & $(32.0)$ & & & \\
\hline \multicolumn{2}{|c|}{ Duration (weeks) } & \multicolumn{2}{|c|}{$11.5 \pm 4.15$} & \multicolumn{2}{|c|}{$12.2 \pm 3.85$} & 0.63 & & 0.52 \\
\hline \multicolumn{2}{|c|}{ Dose $(M \pm S D)$} & \multicolumn{2}{|c|}{$930.2 \pm 36.2$} & \multicolumn{2}{|c|}{$930.0 \pm 33.1$} & 0.01 & & 0.98 \\
\hline
\end{tabular}

Continuous data are represented in mean \pm SD; categorical data are represented in number and percentage. Group I was treated by valproate and group II was treated by levetiracetam

$M \pm S D$ mean \pm standard deviation 
Table 2 Comparison between pre- and post-valproate (group I) treatment regarding sex hormone assay and sperm parameters

\begin{tabular}{|c|c|c|c|}
\hline \multirow[t]{2}{*}{ Variable } & \multicolumn{2}{|c|}{ Valproate (group I) } & \multirow[t]{2}{*}{$P$ value } \\
\hline & Pre-treatment & Post-treatment & \\
\hline \multicolumn{4}{|l|}{ Sex hormones assay } \\
\hline • Luteinizing hormone (LH) & $4.86 \pm 1.8$ & $3.4 \pm 1.37$ & $0.01^{*}$ \\
\hline - Follicle-stimulating hormones (FSH) & $6.0 \pm 2.6$ & $4.6 \pm 2.8$ & $0.01^{*}$ \\
\hline - Prolactin (PRL) & $8.5 \pm 4.0$ & $14.7 \pm 8.4$ & $<0.001^{* *}$ \\
\hline - Testosterone $(\mathrm{T})$ & $5.8 \pm 2.0$ & $5.4 \pm 1.8$ & 0.1 \\
\hline • Estradiol (E2) & $25.4 \pm 11.3$ & $24.6 \pm 10.9$ & 0.9 \\
\hline \multicolumn{4}{|l|}{ Sperm parameters: } \\
\hline - Concentration & $29.9 \pm 12.8$ & $25 \pm 12.1$ & $0.01^{*}$ \\
\hline - Total count & $45.6 \pm 9.0$ & $37.6 \pm 9.1$ & $0.01^{*}$ \\
\hline - Total motility & $58.2 \pm 10.1$ & $45.8 \pm 14.0$ & $0.001^{* *}$ \\
\hline - Normal morphology & $3.4 \pm 1.4$ & $2.88 \pm 1.05$ & $0.029^{*}$ \\
\hline
\end{tabular}

Data are represented in mean \pm SD

*Significant

**Highly significant

of free testosterone and/or albumin-bound testosterone. Also, SHBG is the most important regulator for the biologic effect of the testosterone on the target tissue (it decreases the activity of the testosterone on the target cells). Secondary hormonal changes can occur with epilepsy due to a negative feedback loop between testosterone and pituitary hormone. Disturbances may occur at the levels of the hypothalamic, pituitary, and/or gonadal levels [17].

Also, in our research, we found statistical significant increase in serum prolactin (PRL) level in valproatetreated group at post-treatment checkpoint $(P<0.001)$. This was in agreement with Xiaotian and colleagues [10]. Similarly, in their study, Rabie and colleagues [18] observed that the serum level of prolactin in valproate- treated patients was significantly higher when compared with the control group. Valproate-induced hyperprolactinemia results from altered regulation of GABA-ergic, noradregneric, and serotonergic neurons which in turn modulate dopamine release [10].

Adding to that, seizures induce neuronal discharges which stimulate the hypothalamus and altered prolactin secretion by the pituitary gland [19]. Prolactin levels are further elevated through significantly increased sensitivity to prolactin inhibitory factor and prolactin-releasing factor [10].

Regarding sperm parameters, we found a significant decrease in all measured items at post-treatment checkpoint in the valproate-treated group (group I). This was in going with the results of other studies $[14,10,20]$.

Table 3 Comparison between pre- and post-levetiracetam (group II) treatment regarding sex hormone assay and sperm parameters

\begin{tabular}{|c|c|c|c|}
\hline \multirow[t]{2}{*}{ Variable } & \multicolumn{2}{|c|}{ Levetiracetam (group II) } & \multirow{2}{*}{$\begin{array}{l}P \\
\text { value }\end{array}$} \\
\hline & Pre-treatment & Post-treatment & \\
\hline \multicolumn{4}{|l|}{ Sex hormones assay } \\
\hline - Luteinizing hormone (LH) & $4.7 \pm 2.0$ & $3.7 \pm 1.9$ & 0.07 \\
\hline - Follicle-stimulating hormones (FSH) & $6.4 \pm 3.16$ & $5.1 \pm 3.3$ & 0.11 \\
\hline - Prolactin (PRL) & $8.65 \pm 4.2$ & $8.0 \pm 3.6$ & 0.12 \\
\hline - Testosterone (T) & $5.7 \pm 1.6$ & $5.0 \pm 1.97$ & 0.07 \\
\hline - Estradiol (E2) & $25.1 \pm 11.7$ & $24.6 \pm 11.5$ & 0.6 \\
\hline \multicolumn{4}{|l|}{ Sperm parameters } \\
\hline - Concentration & $29.1 \pm 12.6$ & $23.2 \pm 10.3$ & $0.01^{*}$ \\
\hline - Total count & $45.6 \pm 9.1$ & $38.8 \pm 8.3$ & $0.01^{*}$ \\
\hline - Total motility & $57.96 \pm 10.9$ & $48.1 \pm 12.3$ & $0.001^{* *}$ \\
\hline - Normal morphology & $6.3 \pm 1.8$ & $5.1 \pm 1.5$ & $0.01^{*}$ \\
\hline
\end{tabular}

Data are represented in mean \pm SD

*Significant

**Highly significant 


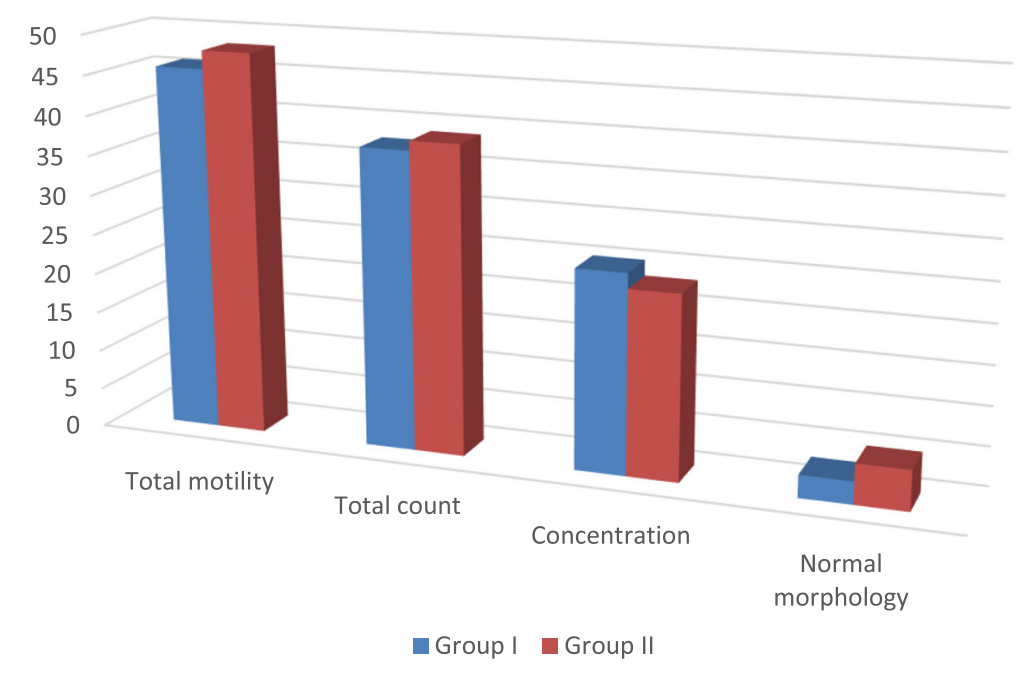

Group I: was treated by valporate; group II: was treated by levetiracetam

Fig. 1 Comparison between group I and group || regarding sperm parameters at post-treatment checkpoint

Free carnitine to total carnitine ratio decrease in male patients with epilepsy treated by VAP, and this may further affect sperm motility and decrease in fertility rates in these patients [14]. Other studies have confirmed that VAP directly affect sperm motility and marked abnormalities of sperm morphology in rats, dogs, and sheep $[20,21]$. In humans treated with VPA, the number of motile sperms is reduced and several morphological changes occurred. These morphological changes increased sperm tail pathology which was suggested to be a cause of decreased of males' fertility [7]. In in vitro research, it has been proposed that VPA directly affects sperm motility by inducing membrane stabilization [13].

Regarding levetiracetam-treated group (group II), we found non-significant difference between pre- and postcheckpoint of male sex hormones. This result was in agreement with two previous studies $[2,10]$. That is, due to the main metabolic pathway of LEV through hydrolase acetyl animation transformation, the main metabolite (UCB 1057) is not transformed by liver cytochromes and LEV and its main metabolites do not affect the activity of hepatic cytochromes p450 [10].

In Svalheim and colleagues' [22] study, free testosterone and rostenedione levels have been found to be elevated in patients treated with LEV compared with those patients from the control group. However, the same elevation was also recorded in the carbamazepine and lamotrigine groups. The authors have attributed these changes to the disease itself [22]. Harden and colleagues, 2010 [23], found increased testosterone levels in eight men after LEV treatment; however, this study was uncontrolled and was included in a very heterogeneous group of patients.
We found significant decrease in all sperm parameters among LEV-treated group at post-treatment checkpoint. This was according to two previous studies $[2,10]$. Sperm parameter changes without alteration of sex hormone levels suggest a possible peripheral mechanism of adverse action [2].

Moreover, in another animal study, LEV adversely affected sperm parameters by reducing sperm concentration and motility, increasing abnormal sperm morphology and sperm DNA damage, and causing damage in the testicular structure of male rats. It is suggested that the mechanism of LEV toxicity in the male reproductive system arises from the promotion of oxidative stress and alterations in hormonal status [24].

Surges and colleagues, 2008 [6], reported that one of the LEV mechanism as antiepileptic drug is its effect on GABA-ergic system as it was associated with GABA-A receptor modification. GABA-A receptors were also found in the testes as well as CNS. It has been shown that GABA-ergic signaling reduced the proliferation of spermatogonial stem cells of the seminiferous tubules [25].

Another explanation of peripheral interference of LEV is the synaptic vesicular protein (SV2A) receptors, which are the most frequently presented form of synaptic vesicles in the glycoprotein family. These receptors were widely present in the CNS and endocrine cells [26]. The peripheral presence of such receptors in the testes could be the cause of reduced sperm parameters among LEV-treated groups.

Our study has some strength, as the pre- and postantiepileptic drug treatment serum sex hormone assay and semen analysis were done for the same patients (self-control groups). We think that will nullify various external factors. The second strength is that we assessed 
newly diagnosed epileptic males. Thus, we were able to exclude factors associated with chronicity of the disease and the effects of previously administered AEDs.

Our study has some limitations. First of all, this study group is relatively small. Also, we assessed only the early effects of these drugs on the reproductive function of these epileptic patients.

\section{Conclusion and recommendation}

Levetiracetam is unlikely to cause the type of reproductive system damage that can occur during valproate metabolism which can induce a series of reproductive endocrine changes. Good tailoring of antiepileptic drugs for male epileptic patients is to minimize probable suffering of these patients and avoid a major impact on the quality of their life. We recommend further studies on a larger group of population and longer follow-up period to explore the risks and mechanisms of such treatmentrelated side effects on reproductive functions, particularly in newly diagnosed epileptic males.

\section{Authors' contributions}

WM, $\mathrm{RN}, \mathrm{NH}$, and $\mathrm{AO}$ carried out this work. WM designed the study, had done the statistical analysis, and wrote the manuscript. WM and RN did the literature search and coordinated the research team. WM, RN, and NH collected the patients, gathered the clinical data, and reviewed the manuscript. AO helped the laboratory work of the study. All authors were involved in drafting the article or revising it critically for important intellectual content, and all authors approved the final version to be published.

\section{Funding}

There is no source of funding for the research.

\section{Availability of data and materials}

Supporting the results of this article is included within the article (and its additional file(s)).

\section{Ethics approval and consent to participate}

The study was approved from the Institutional Ethics Committee of Faculty of Medicine, Zagazig University (ZU-IRB\#4731 24-1-2018). A written consent was taken from all of the participants after explaining the details, benefits, and risks to them.

\section{Consent for publication}

This has been obtained from the participants involved in the study to report their individual patient data.

\section{Competing interests}

The authors declare that they have no competing interests.

\section{Author details}

'Department of Neurology, Faculty of Medicine, Zagazig University, Zagazig, Sharkia, Egypt. ${ }^{2}$ Department of Clinical Pathology, Faculty of Medicine, Zagazig University, Zagazig, Sharkia, Egypt.

Received: 12 March 2018 Accepted: 23 May 2019

Published online: 10 June 2019

\section{References}

1. Harden CL. Sexual dysfunction in women with epilepsy. Seizure. 2008;17(2): $131-5$.

2. Ceylan M, Yalcin A, Bayraktutan OF, Karabulut I, Sonkaya AR. Effects of levetiracetam monotherapy on sperm parameters and sex hormones: data from newly diagnosed epilepsy patients. Seizure. 2016;41:70-4.

3. Montouris G, Morris GL. Reproductive and sexual dysfunction in men with epilepsy. Epilepsy Behav. 2005;7(2):7-14.
4. Atif M, Sarwar MR, Scahil S. The relationship between epilepsy and sexual dysfunction: a review of the literature. SpringerPlus. 2016;5(1):2070.

5. Johannessen CU, Johannessen SI. Valproate: past, present, and future. CNS Drug Rev. 2003:9(2):199-216.

6. Surges R, Volynski KE, Walker MC. Is levetiracetam different from other antiepileptic drugs/levetiracetam and its cellular mechanism of action in epilepsy revisited. Ther Adv Neurol Disord. 2008;1 (1):13-24.

7. Svalheim S, Sveberg L, Mochol M, Taubøll E. Interactions between antiepileptic drugs and hormones. Seizure. 2015;28:12-7.

8. Berg AT, Berkovic SF, Brodie MJ, Buchhalter J, Cross JH, van Emde Boas W, et al. Revised terminology and concepts for organization of seizures and epilepsies: report of the ILAE Commission on Classification and Terminology, 2005-2009. Epilepsia. 2010;51(4):676-85.

9. CDC. Sample size reference: Epi info 7 (7.1.5.2). In: The division of surveillance and epidemiology. Atlanta: Centers for Disease control and Prevention; 2015. Available from: https://wwwn.cdc.gov/epiinfo/7/index.htm.

10. Xiaotian X, Hengzhong Z, Yao X, Zhipan Z, Daoliang X, Yume W. Effects of antiepileptic drugs on reproductive endocrine function, sexual function and sperm parameters in Chinese Han men with epilepsy. J Clin Neurosci. 2013;20(11):1492-7.

11. Levesque R. SPSS programming and data management: a guide for SPSS and SAS users. 4th ed Edition. Chicago: SPSS Inc; 2007.

12. Kose-Ozlece H, llık F, Cecen K, Huseyınoglu N, Serım A. Alterations in semen parameters in men with epilepsy treated with valproate. Iran J Neuro. 2015; 14(3):164-7.

13. Rattya J, Turkka J, Pakarinen AJ, Knip M, Kotila MA, Lukkarinen O, et al. Reproductive effects of valproate, carbamazepine and oxcarbazepine in men with epilepsy. Neurology. 2001;56:31-6.

14. Roste LS, Tauboll E, Morkrid L, Bjørnenak T, Saetre ER, Mørland T, et al. Antiepileptic drugs alter reproductive endocrine hormones in men with epilepsy. Eur J Neurol. 2005;12(2):118-24.

15. Mikkonen K, Tapanainen P, Pakarinen AJ, Päivänsalo M, Isojärvi II, Vainionpää $L K$, et al. Serum androgen levels and testicular structure during pubertal maturation in male subjects with epilepsy. Epilepsia. 2004;45(7):769-76.

16. Najafi MR, Ansari B, Zare M, Fatehi F, Sonbolestan A. Effects of antiepileptic drugs on sexual function and reproductive hormones of male epileptic patients. Iran J Neurol. 2012:11(2):37-41.

17. Hamed SA, Ahmad HK, Youssef AH, Metwaly NA, Hassan MM, Mohamad HO. Erectile function in men with epilepsy: relationship to demographic, clinical endocrinal, psychosocial-related variables. J Neurol Neurosci. 2013; 4(2). https://doi.org/10.3823/337.

18. Rabie MO, El-din ESA, Rashed KH, Bahnasy WS, El-Serogy HA. A study on serum levels of testosterone and prolactin hormones in male epileptic adolescents. Egypt J Neurol Psychiatry Neurosurg. 2016;53:79-83.

19. Herzog AG, Coleman AE, Jacobs AR, Klein P, Friedman MN, Drislane FW, et al. Interictal EEG discharges, reproductive hormones and menstrual disorders in epilepsy. Ann Neurol. 2003;54(5):625-37.

20. Isojarvi JI, Lofgren E, Juntunen KST, Pakarinen AJ, Päivänsalo M, Rautakorpi I, et al. Effect of epilepsy and antiepileptic drugs on male reproductive health. Neurology. 2004;62(2):247-53.

21. Chen L, Feng P, Wang J, Liu L, Zhou D. Intravenous sodium valproate in mainland China for the treatment of diazepam refractory convulsive status epilepticus. J Clin Neurosci. 2009;16(4):524-6.

22. Svalheim S, Tauboll E, Luef G, Lossius A, Rauchenzauner M, Sandvand F, et al. Differential effects of levetiracetam, carbamazepine, and lamotrigine on reproductive endocrine function in adults. Epilepsy Behav. 2009;16(2):281-7.

23. Harden CL, Nikolov BG, Kandula P, Labar DR, Pannullo S. Effect of levetiracetam on testosterone levels in male patients. Epilepsia. 2010;51(11):2348-51.

24. Baysal M, Ilgin S, Kilic G, Kilic V, Ucarcan S, Atli O. Reproductive toxicity after levetiracetam administration in male rats: evidence for role of hormonal status and oxidative stress. PLoS One. 2017:12(4):e0175990.

25. Du Y, Du Z, Zheng H, Wang D, Li S, Yan Y, et al. GABA exists as a negative regulator of cell proliferation in spermatogonial stem cells. Cell Mol Biol Lett. 2003;18(2):149-62.

26. Wood MD, Gillard M. Evidence for a differential interaction of brivaracetam and levetiracetam with the synaptic vesicle 2A protein. Epilepsia. 2017;58(2):255-62

\section{Publisher's Note}

Springer Nature remains neutral with regard to jurisdictional claims in published maps and institutional affiliations. 